\title{
Direct Block Predictor-Corrector Method for the Solution of General Fourth Order Odes
}

\author{
Olabode B. T. ${ }^{1} \&$ Alabi T. J. ${ }^{2}$ \\ ${ }^{1}$ Department of Mathematical Sciences, Federal University of Technology, Akure, Nigeria \\ ${ }^{2}$ Department of Mathematics/Statistics and Computer Science, Kogi State Polythenic, Lokoja \\ Correspondence: Olabode B. T., Department of Mathematical Sciences, Federal University of Technology, Akure, \\ Nigeria. E-mail: olabodebola@yahoo.com
}

Received: June 2, 2012 Accepted: September 5, 2012 Online Published: January 8, 2013

doi:10.5539/jmr.v5n1p26 URL: http://dx.doi.org/10.5539/jmr.v5n1p26

\begin{abstract}
This article presented the direct block predictor-corrector method for solving general higher order initial value problems of ordinary differential equation. Method of collocation and interpolation of power series approximate solution was used to derive a continuous linear multistep method. Block method was later used to generate the non overlapping solution at selected grid points. The method developed, is self starting, consistent, symmetric, zero-stable and convergent. The performance of the new block method was tested with some fourth order initial value problems and it was found to compare favorably with the existing methods.
\end{abstract}

Keywords: Linear Multistep Methods (LMMs), fourth order, Initial Value Problems (IVPs), Ordinary Differential Equations (ODEs), interval of periodicity, predictor-corrector (P-C)

\section{Introduction}

Consider the higher order initial value problems of ordinary differential equations of the form;

$$
y^{n}=f\left(x, y, y^{\prime}, y^{\prime \prime}, \cdots, y^{n-1}\right), \quad y(a)=y_{0}, \quad y^{i}(a)=y_{i}, \quad i=1, \ldots, n-1, \quad n \geq 4 .
$$

This class of problems has a lot of applications in science and engineering especially in mechanical systems without dissipation, control theory and celestial mechanics. Conventionally, Equation (1.1) is usually reduced to systems of first order differential equations before an approximate method is applied to solve it (Lambert, 1973; Fatunla, 1988).

Direct method of solving higher order ordinary differential equations in predictor-corrector mode have been studied by many scholars including: Awoyemi (2003), Adee et al. (2005), Awoyemi and Idowu (2005), Fatunla (1994), Kayode (2009), Awoyemi and Kayode (2005) to mention but a few. Awoyemi (2003) investigated a p-stable linear multistep method for general third order initial value problems of ordinary differential equations. Awoyemi and Idowu (2005) developed a class of hybrid collocation method for third order ordinary differential equations. These authors respectively proposed some linear multistep methods with continuous coefficients using Taylor series expansion to supply the starting values. These methods were implemented in predictor-corrector mode, although they yielded good results but the implementation is too costly, because the predictors are developed in the same way as the correctors and subroutines are very complicative to write since they require special techniques to supply the starting values. This eventually leads to longer computer time and human effort. In order to circumvent the set backs of the predictor-corrector methods, this article therefore proposes the direct block predictor-corrector method for solving general fourth order initial value problems of ordinary differential equation. This block method is capable of giving evaluations at different grids points without overlapping as in the predictor-corrector method, hence does not require the development of separate predictors, starting values. The method is consistent, symmetric, zero-stable and it is more accurate than the existing methods.

Definition 1.1 The block method is said to be stable if as $h \rightarrow 0$ the roots $r_{j}, \mathrm{j}=1,2, \ldots, \mathrm{k}$ of the first characteristic polynomial $\rho(R)=0$, that is $\rho(R)=\operatorname{det}\left[\sum A^{i} R^{k-1}\right]=0$, satisfy $|R| \leq 1$ and for these roots with $|R| \leq 1$ must have multiplicity equal to unity.

Definition 1.2 If the block method be $R \times R$ matrix, then, it is zero-stable, if as $h^{\mu} \rightarrow 0,\left|R A^{0}-A^{i}\right|=R^{r-\mu}(R-1)=0$. For those root with $\left|R_{j}\right| \leq 1$, the multiplicity must not exceed the order of the differential equation (Fatunla 1991). 
In recent time, authors have adopted the block method for solving higher order ordinary differential equations. Among them are Jator (2007), Jator and Li (2009), Omar and Suleiman (2009), Badmus and Yahaya (2009), Olabode (2009a \& b), Olabode and Yusuph (2009), Siamak (2010), Zarina et al. (2009) and Awoyemi et al. (2011). In Zanariah et al. (2012) a two-point four step direct implicit block method of order 7 for solving third order ordinary differential equations using variable step size strategy was proposed. In this paper, the works of Shampine and Watt (1969) and Abbas (2006) were adopted. They gave the general discrete block formula as:

$$
A^{(0)} Y_{m}=e y_{n}+h d f\left(y_{n}\right)+h^{\mu} B f\left(Y_{m}^{0}\right)
$$

where the prediction equation is given by

$$
Y_{m}^{0}=e y_{n}+h^{\mu} d f\left(y_{n}\right)
$$

Putting (1.3) into (1.2) one obtains

$$
A^{(0)} Y_{m}=e y_{n}+h d f\left(y_{n}\right)+h^{\mu} B f\left(e y_{n}+h^{\mu} d f\left(y_{n}\right)\right)
$$

According to Abbas (2006), (1.4) is called block predictor-corrector method and self-starting since the prediction equation is obtained directly from the general block formula (1.2).

\section{Method}

Following Abbas (2006), the general discrete block formula is as follow:

$$
A^{(0)} Y_{m}=e y_{n}+h d f\left(y_{n}\right)+h^{\mu} B f\left(Y_{m}^{0}\right)
$$

where $e$ and $d$ are $R$-vectors, $A^{0}$ and $B$ are $R \times R$ identity matrices, $f$ is also a $R$-vectors whose $j$ th entry is $f_{n+j}=f\left(x_{n+j} ; y_{n+j}\right) ; 1<j<R$. Equation (2.1) is implicit in $Y_{m}$ and it has to be solved iteratively by predicted solution values. A predictor equation for $Y_{m}$ can be expressed in the form:

$$
Y_{m}^{0}=e y_{n}+\sum_{\lambda=0}^{m} h^{\mu+\lambda} f^{\lambda}\left(y_{n}\right)
$$

where $f^{\lambda}\left(y_{n}\right)=\frac{\partial^{\lambda}}{\partial x^{\lambda}} f\left(x, y, y^{\prime}, y^{\prime \prime}, y^{\prime \prime \prime}\right)$. Putting (2.2) into (1.2) one obtains

$$
A^{(0)} Y_{m}=e y_{n}+h d f\left(y_{n}\right)+h^{\mu} B f\left(e y_{n}+\sum_{\lambda=0}^{m} h^{\mu+\lambda} f^{\lambda}\left(y_{n}\right)\right) .
$$

where $\mu$ is the order of the differential Equation (1.1) and $m=4$ in this work. Equation (2.3) is the new block predictor-corrector method. Moreover, consider a monomial power series of the form:

$$
P(x)=\sum_{j=0}^{r+s-1} a_{j} x^{j}
$$

$r$ is the interpolation point and $s$ is the collocation point. Equation (2.4) is used as the basis or trial function to provide the approximate solution in the form:

$$
y(x)=\sum_{j=0}^{r+s-1} a_{j} x^{j}
$$

$a_{j} \in R, j=0,1,2, \ldots, r+s-1, y \in C^{m},(a, b) \subset P(x)$, whose higher derivatives are

$$
\begin{gathered}
y^{\prime}(x)=\sum_{j=0}^{r+s-1} j a_{j} x^{j-1} \\
\left.y^{\prime \prime}(x)=\sum_{j=0}^{r+s-1} j(j-1)\right) a_{j} x^{j-2}
\end{gathered}
$$




$$
\begin{gathered}
y^{\prime \prime \prime}(x)=\sum_{j=0}^{r+s-1} j(j-1)(j-2) a_{j} x^{j-3} \\
y^{\prime \prime \prime \prime}(x)=\sum_{j=0}^{r+s-1} j(j-1)(j-2)(j-3) a_{j} x^{j-4}
\end{gathered}
$$

substituting (2.9) into (1.1) one obtains

$$
f\left(x, y, y^{\prime}, y^{\prime \prime}, y^{\prime \prime \prime}\right)=\sum_{j=0}^{r+s-1} j(j-1)(j-2)(j-3) a_{j} x^{j-4}
$$

Interpolating (2.5) at $x=x_{n+j}, j=0,1,2, \ldots, k-1$ and collocating (2.10) at $x=x_{n+j}, j=0,1,2, \ldots, k$ one obtains a system of equations which after solving for $a_{j}^{\prime} s$ and substituting into (2.5), with some manipulation yields, a linear multistep method with continuous coefficients in the form:

$$
y(x)=\sum_{j=0}^{r} \alpha_{j}(x) y_{n+j}+h^{4} \sum_{j=0}^{s} \beta_{j}(x) f_{n+j}
$$

The coefficients $\alpha_{j}(x)$ and $\beta_{j}(x)$ are expressed as function of $t=\frac{x-x_{n+3}}{h}$ as follows:

$$
\begin{aligned}
& \alpha_{0}(t)=-\frac{1}{3}\left(32 t^{3}-48 t^{2}+22 t-3\right) \\
& \alpha_{1}(t)=32 t^{3}-40 t^{2}+12 t \\
& \alpha_{2}(t)=-32 t^{3}+32 t^{2}-6 t \\
& \alpha_{3}(t)=\frac{1}{3}\left(32 t^{3}-24 t^{2}+4 t\right) \\
& \beta_{0}(t)=\frac{h^{4}}{15120}\left(24576 t^{8}-122880 t^{7}+250880 t^{6}-268800 t^{5}+161280 t^{4}-53424 t^{3}+8926 t^{2}-579 t\right) \\
& \beta_{1}(t)=\frac{h^{4}}{3780}\left(24576 t^{8}-1105924 t^{7}+186368 t^{6}-129024 t^{5}+46368 t^{3}-21062 t^{2}+2715 t\right) \\
& \beta_{2}(t)=\frac{h^{4}}{2520}\left(24576 t^{8}-98304 t^{7}+136192 t^{6}-64512 t^{5}+1680 t^{3}+2654 t^{2}-627 t\right) \\
& \beta_{3}(t)=\frac{h^{4}}{3780}\left(24576 t^{8}-86016 t^{7}+100352 t^{6}-43008 t^{5}+4032 t^{3}-566 t^{2}+21 t\right) \\
& \beta_{4}(t)=\frac{h^{4}}{15120}\left(24576 t^{8}-73728 t^{7}+78848 t^{6}-32256 t^{5}+3024 t^{3}-482 t^{2}-3 t\right) .
\end{aligned}
$$

Evaluating (2.12) at $t=1$ gives the discrete method

$$
y_{n+4}-4 y_{n+3}+6 y_{n+2}-4 y_{n+1}+y_{n}=\frac{h^{4}}{720}\left(-f_{n+4}+124 f_{n+3}+474 f_{n+2}+124 f_{n+1}-f_{n}\right) .
$$

Equation (2.13) is of order seven, symmetric, consistent and zero-stable. The first, second and the third derivatives of (2.12) were obtained.

\subsection{Impelementation of the Block Method}

In this section, we present the implementation strategy of our work. Differentiating (2.12) and evaluating it, at $t=0(1) 4$ we obtain the following

$$
\begin{aligned}
& h y_{n}^{\prime}=-\frac{11}{6} y_{n}+3 y_{n+1}-\frac{3}{2} y_{n+2}+\frac{1}{3} y_{n+3}+h^{4}\left(-\frac{193}{20160} f_{n}-\frac{181}{1008} f_{n+1}-\frac{209}{3360} f_{n+2}+\frac{1}{720} f_{n+3}-\frac{1}{20160} f_{n+4}\right) \\
& h y_{n+1}^{\prime}=-\frac{1}{3} y_{n}-\frac{1}{2} 3 y_{n+1}+y_{n+2}-\frac{1}{6} y_{n+3}+h^{4}\left(-\frac{31}{6048} f_{n}-\frac{727}{15120} f_{n+1}+\frac{397}{10080} f_{n+2}-\frac{13}{3024} f_{n+3}+\frac{41}{60480} f_{n+4}\right) \\
& h y_{n+2}^{\prime}=\frac{1}{6} y_{n}-y_{n+1}+\frac{1}{2} y_{n+2}+\frac{1}{3} y_{n+3}+h^{4}\left(\frac{11}{12096} f_{n}-\frac{493}{15120} f_{n+1}-\frac{79}{1440} f_{n+2}+\frac{59}{15120} f_{n+3}-\frac{41}{60480} f_{n+4}\right) \\
& h y_{n+3}^{\prime}=-\frac{1}{3} y_{n}+\frac{3}{2} y_{n+1}-3 y_{n+2}+\frac{11}{6} y_{n+3}+h^{4}\left(-\frac{23}{20160} f_{n}-\frac{311}{5040} f_{n+1}+\frac{121}{672} f_{n+2}+\frac{47}{5040} f_{n+3}+\frac{1}{20160} f_{n+4}\right)
\end{aligned}
$$




$$
h y_{n+4}^{\prime}=-\frac{11}{6} y_{n}+7 y_{n+1}-\frac{19}{2} y_{n+2}+\frac{13}{3} y_{n+3}+h^{4}\left(-\frac{151}{60480} f_{n}+\frac{679}{2100} f_{n+1}+\frac{12793}{10080} f_{n+2}+\frac{7489}{15120} f_{n+3}+\frac{85}{12096} f_{n+4}\right)
$$

Evaluating the second derivative of (2.12) at $t=0(1) 4$ we obtain the following

$$
\begin{gathered}
h^{2} y_{n}^{\prime \prime}=2 y_{n}-5 y_{n+1}+4 y_{n+2}-y_{n+3}+h^{4}\left(\frac{4463}{60480} f_{n}+\frac{10531}{15120} f_{n+1}+\frac{1327}{10080} f_{n+2}+\frac{283}{15120} f_{n+3}-\frac{241}{60480} f_{n+4}\right) \\
h^{2} y^{\prime \prime}{ }_{n+1}=y_{n}-2 y_{n+1}+y_{n+2}+h^{4}\left(-\frac{157}{60480} f_{n}-\frac{1187}{15120} f_{n+1}-\frac{17}{10080} f_{n+2}-\frac{11}{15120} f_{n+3}+\frac{11}{60480} f_{n+4}\right) \\
h^{2} y_{n+2}^{\prime \prime}=y_{n+1}-2 y_{n+2}+y_{n+3}+h^{4}\left(\frac{11}{60480} f_{n}-\frac{53}{15120} f_{n+1}-\frac{773}{10080} f_{n+2}-\frac{53}{15120} f_{n+3}+\frac{11}{60480} f_{n+4}\right) \\
h^{2} y_{n+3}^{\prime \prime}=-y_{n}+4 y_{n+1}-5 y_{n+2}+2 y_{n+3}+h^{4}\left(-\frac{73}{60480} f_{n}+\frac{2593}{15120} f_{n+1}+\frac{6619}{10080} f_{n+2}+\frac{1417}{15120} f_{n+3}-\frac{241}{60480} f_{n+4}\right) \\
h^{2} y_{n+4}^{\prime \prime}=-2 y_{n}+7 y_{n+1}-8 y_{n+2}+3 y_{n+3}+h^{4}\left(-\frac{409}{60480} f_{n}+\frac{5491}{15120} f_{n+1}+\frac{14599}{10080} f_{n+2}+\frac{15739}{15120} f_{n+3}-\frac{859}{60480} f_{n+4}\right)
\end{gathered}
$$

Evaluating the third derivative of (2.12) at $t=0(1) 4$ gives,

$$
\begin{gathered}
h^{3} y_{n}^{\prime \prime \prime}=-y_{n}+3 y_{n+1}-3 y_{n+2}+y_{n+3}+h^{4}\left(\frac{53}{160} f_{n}-\frac{23}{20} f_{n+1}+\frac{1}{16} f_{n+2}-\frac{1}{10} f_{n+3}+\frac{3}{180} f_{n+4}\right) \\
h^{3} y_{n+1}^{\prime \prime \prime}=-y_{n}+3 y_{n+1}-3 y_{n+2}+y_{n+3}+h^{4}\left(\frac{5}{280} f_{n}-\frac{91}{360} f_{n+1}-\frac{73}{240} f_{n+2}+\frac{17}{360} f_{n+3}-\frac{11}{1440} f_{n+4}\right) \\
h^{3} y^{\prime \prime \prime}{ }_{n+2}=-y_{n}+3 y_{n+1}-3 y_{n+2}+y_{n+3}+h^{4}\left(-\frac{13}{1440} f_{n}+\frac{41}{180} f_{n+1}+\frac{79}{240} f_{n+2}-\frac{1}{18} f_{n+3}+\frac{11}{1440} f_{n+4}\right) \\
h^{3} y^{\prime \prime \prime}{ }_{n+3}=-y_{n}+3 y_{n+1}-3 y_{n+2}+y_{n+3}+h^{4}\left(\frac{1}{160} f_{n}+\frac{1}{8} f_{n+1}+\frac{77}{80} f_{n+2}+\frac{17}{40} f_{n+3}-\frac{3}{160} f_{n+4}\right) \\
h^{3} y^{\prime \prime \prime}{ }_{n+4}=-y_{n}+3 y_{n+1}-3 y_{n+2}+y_{n+3}+h^{4}\left(-\frac{29}{1440} f_{n}+\frac{49}{180} f_{n+1}+\frac{143}{240} f_{n+2}+\frac{119}{90} f_{n+3}+\frac{95}{288} f_{n+4}\right)
\end{gathered}
$$

Putting (2.13), (2.14), (2.19) and (2.24) into (2.28) and simplifying, the coefficients of (1.2) are as follows

$$
\begin{gathered}
E=\left[\begin{array}{llll}
1 & 1 & \frac{1}{2} & \frac{1}{6} \\
1 & 2 & 2 & \frac{4}{3} \\
1 & 3 & \frac{9}{2} & \frac{9}{2} \\
1 & 4 & 8 & \frac{32}{3}
\end{array}\right], D=\left[\begin{array}{cccc}
0 & 0 & 0 & \frac{3373}{120960} \\
0 & 0 & 0 & \frac{296}{945} \\
0 & 0 & 0 & \frac{9}{2} \\
0 & 0 & 0 & \frac{32}{3}
\end{array}\right] \\
A^{(0)}=\left[\begin{array}{llll}
1 & 0 & 0 & 0 \\
0 & 1 & 0 & 0 \\
0 & 0 & 1 & 0 \\
0 & 0 & 0 & 1
\end{array}\right], D=\left[\begin{array}{cccc}
\frac{139}{6048} & -\frac{283}{20160} & \frac{179}{30240} & -\frac{131}{120960} \\
\frac{472}{945} & -\frac{2}{9} & \frac{88}{945} & -\frac{16}{945} \\
\frac{2889}{1120} & -\frac{1539}{2240} & \frac{81}{224} & -\frac{297}{4480} \\
\frac{1024}{135} & -\frac{256}{315} & \frac{1024}{945} & \frac{32}{189}
\end{array}\right]
\end{gathered}
$$

Writing (2.29) explicitly one obtains the following

$$
\begin{gathered}
y_{n+1}=y_{n}+h y_{n}^{\prime}+\frac{1}{2} h^{2} y_{n}^{\prime \prime}+\frac{1}{6} h^{3} y_{n}^{\prime \prime \prime}+h^{4}\left(\frac{3373}{120960} f_{n}-\frac{139}{6048} f_{n+1}-\frac{283}{20160} f_{n+2}+\frac{179}{30240} f_{n+3}-\frac{131}{120960} f_{n+4}\right) \\
y_{n+2}=y_{n}+2 h y_{n}^{\prime}+2 h^{2} y_{n}^{\prime \prime}+\frac{4}{3} h^{3} y_{n}^{\prime \prime \prime}+h^{4}\left(\frac{296}{945} f_{n}+\frac{472}{945} f_{n+1}-\frac{2}{9} f_{n+2}+\frac{88}{945} f_{n+3}-\frac{16}{945} f_{n+4}\right) \\
y_{n+3}=y_{n}+3 h y_{n}^{\prime}+\frac{9}{2} h^{2} y_{n}^{\prime \prime}+\frac{9}{2} h^{3} y_{n}^{\prime \prime \prime}+h^{4}\left(\frac{5319}{4480} f_{n}+\frac{2889}{1120} f_{n+1}-\frac{1539}{2240} f_{n+2}+\frac{81}{224} f_{n+3}-\frac{297}{4480} f_{n+4}\right)
\end{gathered}
$$




$$
y_{n+4}=y_{n}+4 h y_{n}^{\prime}+8 h^{2} y_{n}^{\prime \prime}+\frac{32}{3} h^{3} y_{n}^{\prime \prime \prime}+h^{4}\left(\frac{2816}{945} f_{n}+\frac{1024}{135} f_{n+1}-\frac{256}{315} f_{n+2}+\frac{1024}{945} f_{n+3}-\frac{32}{189} f_{n+4}\right)
$$

Equations (2.30) - (2.33), in block form, are implemented using a MATLAB algorithm, gives solution at selected grid points within the integration interval were obtained.

\subsection{Analysis of the Block Method}

The basic properties of the block method which include the order, zero stability and the convergence of the method was investigated.

\subsubsection{Order of the Method}

The methods proposed by Lambert (1973) and Fatunla (1995) were employed in obtaining the order of the new block method as: $[7,7,7,7]^{T}$ with the error constants $\left[\frac{1469}{1814400}, \frac{176}{14175}, \frac{1107}{22400}, \frac{256}{2025}\right]^{T}$.

2.2.2 Zero Stability of the Block Method

For the new method

$$
\begin{gathered}
A=\operatorname{det}\left[z\left(\begin{array}{llll}
1 & 0 & 0 & 0 \\
0 & 1 & 0 & 0 \\
0 & 0 & 1 & 0 \\
0 & 0 & 0 & 1
\end{array}\right)-\left(\begin{array}{llll}
0 & 0 & 0 & 1 \\
0 & 0 & 0 & 1 \\
0 & 0 & 0 & 1 \\
0 & 0 & 0 & 1
\end{array}\right)\right]=0 \\
A=z^{4}-z^{3}=0, z=0,0,0,1
\end{gathered}
$$

which implies zero stability (see Definition 1.2). The block method is also consistent, as it has the order $p$ greater than 1. Hence the convergence of the method is asserted (Henrici, 1962).

\subsubsection{Region of Absolute Stability}

In this article, the Locus method was used to determine the region of absolute stability. The boundary locus method is given by

$$
h(\theta)=\frac{\rho\left(e^{i \theta}\right)}{\sigma\left(e^{i \theta}\right)}=\frac{\rho(r)}{\sigma(r)}
$$

Where $\rho(r)$ and $\sigma(r)$ are the first and second characteristics polynomial respectively as

$$
r=e^{i \theta}=\cos \theta+i \sin \theta
$$

Applying (2.35) and (2.36) to the derived scheme, we have

$$
\begin{gathered}
h(r)=\frac{r^{4}-4 r^{3}+6 r^{2}-4 r+1}{\frac{1}{720}\left(-r^{4}+124 r^{3}+474 r^{2}+124 r-1\right)} \\
h(\theta)=\frac{(\cos 4 \theta-4 \cos 3 \theta+6 \cos 2 \theta-4 \cos \theta+1)+i(\sin 4 \theta-4 \sin 3 \theta+6 \sin 2 \theta-4 \sin \theta)}{\frac{1}{720}[(-\cos 4 \theta+124 \cos 3 \theta+474 \cos 2 \theta+124 \cos \theta-1)+i(-\sin 4 \theta+124 \sin 3 \theta+474 \sin 2 \theta+124 \sin \theta]}
\end{gathered}
$$

Multiplying by conjugate, considering the real part and evaluating at intervals of $\left(0^{0}, 180^{\circ}\right)$ we have $\left(0^{0}, 9.52^{0}\right)$.

\section{Numerical Experiments, Results and Discussion}

This section deals with numerical experiments and results, using the algorithm proposed for fourth order ordinary differential equations.

\section{Problem 3.1}

$$
\begin{aligned}
& y^{\prime \prime \prime \prime}+y^{\prime \prime}=0,0 \leq \mathrm{x} \leq \frac{\pi}{2}, \mathrm{y}(0)=0, y^{\prime}(0)=\frac{1.1}{72-50 \pi}, \\
& y^{\prime \prime}(0)=-\frac{1}{144-100 \pi}, y^{\prime \prime \prime}(0)=\frac{1.2}{144-100 \pi}, h=\frac{1}{320}
\end{aligned}
$$

Exact solution: $y(x)=\frac{1-x-\cos x-1.2 \sin x}{144-100 \pi}$. 


\section{Problem 3.2}

$$
\begin{gathered}
y^{\prime \prime \prime \prime}=\frac{-\left(8+25 x+30 x^{2}+12 x^{3}+x^{4}\right)}{\left(1+x^{2}\right)} \\
y(0)=0, y^{\prime}(0)=1, y^{\prime \prime}(0)=0, y^{\prime \prime \prime}(0)=-3, h=\frac{1}{320}
\end{gathered}
$$

Exact solution: $y(x)=x(1-x) e^{x}$

Problem 3.3

$$
\begin{gathered}
y^{\prime \prime \prime \prime}=\left(y^{\prime}\right)^{2}-y y^{\prime \prime \prime}-4 x^{2}+e^{x}\left(1-4 x+x^{2}\right) ; ; 0 \leq x \leq 1, \\
y(0)=1, y^{\prime}(0)=, y^{\prime \prime}(0)=3, y^{\prime \prime \prime}=1, h=\frac{1}{320}
\end{gathered}
$$

Exact solution $y(x)=x^{2}+e^{x}$

Table 3.1. Accuracy comparison of the new method with Kayode (2008), $h=1 / 320$

\begin{tabular}{lllll}
\hline $\mathrm{X}$ & $\mathrm{y}$-Exact & y-Approximate & $\begin{array}{l}\text { Error in } \\
\text { method }(\mathrm{k}=4)\end{array}$ & $\begin{array}{l}\text { New } \\
(2008)(\mathrm{k}=4)\end{array}$ \\
\hline 0.103125 & $0.13007993 \mathrm{D}-02$ & $0.13007996 \mathrm{D}-02$ & $0.2168404 \mathrm{D}-020$ & $0.4987329 \mathrm{D}-15$ \\
0.206250 & $0.25317732 \mathrm{D}-02$ & $0.25317737 \mathrm{D}-02$ & $0.4336809 \mathrm{D}-020$ & $0.6765210 \mathrm{D}-15$ \\
0.306250 & $0.36524788 \mathrm{D}-02$ & $0.36524790 \mathrm{D}-02$ & $0.13001043 \mathrm{D}-19$ & $0.3135079 \mathrm{D}-14$ \\
0.406250 & $0.46959532 \mathrm{D}-02$ & $0.46959532 \mathrm{D}-02$ & $0.2602085 \mathrm{D}-019$ & $0.9436028 \mathrm{D}-14$ \\
0.506250 & $0.56576413 \mathrm{D}-02$ & $0.56576424 \mathrm{D}-02$ & $0.1734723 \mathrm{D}-019$ & $0.2211685 \mathrm{D}-13$ \\
0.603125 & $0.65077534 \mathrm{D}-02$ & $0.65077576 \mathrm{D}-02$ & $0.8673617 \mathrm{D}-020$ & $0.4337936 \mathrm{D}-13$ \\
0.703125 & $0.72983134 \mathrm{D}-02$ & $0.72983147 \mathrm{D}-02$ & $0.2602085 \mathrm{D}-019$ & $0.7787086 \mathrm{D}-13$ \\
0.803125 & $0.79985187 \mathrm{D}-02$ & $0.79985202 \mathrm{D}-02$ & $0.5204170 \mathrm{D}-019$ & $0.1286349 \mathrm{D}-12$ \\
0.903125 & $0.86072451 \mathrm{D}-02$ & $0.86072467 \mathrm{D}-02$ & $0.1214306 \mathrm{D}-018$ & $0.1992711 \mathrm{D}-12$ \\
1.003125 & $0.91242822 \mathrm{D}-02$ & $0.91242840 \mathrm{D}-02$ & $0.1734723 \mathrm{D}-018$ & $0.2932324 \mathrm{D}-12$ \\
\hline
\end{tabular}

Table 3.1 shows that, the maximum absolute error of the new block predictor -corrector method of the problem 1 is $0.2168404 D$ - 20 while the maximum absolute error of Kayode (2008) is $0.4987329 D$ - 15 for the same Problem 3.1 which implies that they compare favorably. Both methods are of the same step $k=4$ and were developed for solving fourth order ODEs.

Table 3.2. The y-Exact, $y$-Approximate and error in of Problem 3.2, with $\mathrm{h}=1 / 320$

\begin{tabular}{llll}
\hline$X$ & $y$-Exact & y-Approximate & $\begin{array}{l}\text { Error of the New } \\
\text { Block method }\end{array}$ \\
\hline 0.0031 & 0.0031249847093845 & 0.0031249847094044 & $1.990205 \mathrm{E}-014$ \\
0.0063 & 0.0062498774198671 & 0.0062498774205050 & $6.379298 \mathrm{E}-013$ \\
0.0094 & 0.0093745854286995 & 0.0093745854335519 & $4.852393 \mathrm{E}-012$ \\
0.0125 & 0.0124990152612047 & 0.0124990152816868 & $2.048206 \mathrm{E}-011$ \\
0.0156 & 0.0156230726662503 & 0.0156230727288605 & $6.261025 \mathrm{E}-011$ \\
0.0188 & 0.0187466626116994 & 0.0187466627677537 & $1.560543 \mathrm{E}-010$ \\
0.0219 & 0.0218696892798386 & 0.0218696896176985 & $3.378600 \mathrm{E}-010$ \\
0.0250 & 0.0249920560627830 & 0.0249920567226018 & $6.598189 \mathrm{E}-010$ \\
0.0281 & 0.0281136655578585 & 0.0281136667488685 & $1.191010 \mathrm{E}-009$ \\
0.0313 & 0.0312344195629611 & 0.0312344215833281 & $2.020367 \mathrm{E}-009$ \\
\hline
\end{tabular}

In Table 3.2, $y$-exact, the approximate value and the error of the new block predictor-corrector method for Problem 3.2 are shown. 
Table 3.3. The y-Exact, y-Approximate and error in of Problem 3, with $\mathrm{h}=1 / 320$

\begin{tabular}{llll}
\hline $\mathrm{X}$ & $\mathrm{y}$-Exact & $\mathrm{y}$-Approximate & Error in New method \\
\hline 0.103125 & 1.1192647447875919 & 1.1192647447875916 & $2.220446 \mathrm{E}-016$ \\
0.206250 & 1.2715994931980485 & 1.2715994931980492 & $6.661338 \mathrm{E}-016$ \\
0.306250 & 1.4521109070650133 & 1.4521109070650140 & $6.661338 \mathrm{E}-016$ \\
0.406250 & 1.6662168625001232 & 1.6662168625001246 & $1.332268 \mathrm{E}-015$ \\
0.506250 & 1.9153471099209172 & 1.9153471099209194 & $2.220446 \mathrm{E}-015$ \\
0.603125 & 2.1915815936062044 & 2.1915815936062075 & $3.108624 \mathrm{E}-015$ \\
0.703125 & 2.5144402933336951 & 2.5144402933337013 & $6.217249 \mathrm{E}-015$ \\
0.803125 & 2.8775163877466037 & 2.8775163877466143 & $1.065814 \mathrm{E}-014$ \\
0.903125 & 3.2829361588050943 & 3.2829361588051129 & $1.865175 \mathrm{E}-014$ \\
1.003125 & 3.7330495114951674 & 3.7330495114951967 & $2.930989 \mathrm{E}-014$ \\
\hline
\end{tabular}

Table 3.3 shows y-exact, the approximate value and the error of the new block predictor-corrector method for Problem 3.3.

\section{Conclusion}

In this paper, a new block method for the direct solution of general higher order ordinary differential equations which is better than the conventional method has been presented. Also, the methods derived were analyzed and found to be consistent, zero-stable and convergent. The numerical experiments were given and the results obtained were compared with Kayode (2008) and were found to be better in accuracy than the existing methods.

\section{References}

Abbas, S. (2006). Derivation of a new block method similar to the block trapezoidal rule for the numerical solution of first order IVP's. Science Echoes, 2, 10-24.

Adee, S. O., Onumanyi, P., Sirisena, U. W., \& Yahaya, Y. A. (2005). Note on starting Numererov method Mre Accuratelly By Hybrid Formula of order four for an Initial value problem. Journal of Computationalo and Applied Matrhematics, 175, 369-373.

Awoyemi, D. O. (2003). A p-stable linear multistep method for solving third order ordinary differential equations. Inter. J. Computer Math., 80(8), 985-991. http://dx.doi.org/10.1080/0020716031000079572

Awoyemi, D. O, Adebile E. A., Adesanya, A. O., \& Anake, T. A. (2011). Modified block method for the direct solution of second order ordinary differential equation. Intern. J. of Applied Mathematics and Computation, 3(3), 181-188.

Awoyemi, D. O., \& Idowu. (2005). A Class hybrid collocation methods for third order of ordinary differential equation. Int. J. Comput. Mathe., 82, 1287-1293.

Awoyemi, D. O., \& Kayode, S. J. (2005). A maximal order collocation Method for initial value problems of General Second order ordinary differential equation, Proceedings of the Conference organized by the National Mathematical Centre, Abuja, Nigeria.

Fatunla, S. O. (1988). Numerical methods for initial value problems in ordinary differential equation. New York: Academic Press Inc. Harcourt Brace Jovanovich publisher.

Fatunla, S. O. (1991). Block method for second order ordinary differential equation. International Journal of Computer Mathematics, 41(1 \& 2), 55-63.

Fatunla, S. O. (1994). A class of block methods for second order IVPs. Int. J. Comput. Math., 55, 119-133.

Fatunla, S. O. (1995). A class of block method for second order initial value problems. International Journal of Computer Mathematics, 55(1 \& 2), 119-133. http://dx.doi.org/10.2307/2006091

Henrici, P. (1962). Discrete Variable Methods for ODEs. New York, USA: John Wiley. http://dx.doi.org/10.1002/zamm.19660460521

Jator, S. N. (2007). A sixth order linear multistep method for the direct solution of second order initial value 
problems. International Journal of Pure and Applied Mathematics, 40(1), 457-472.

Jator, S. N., \& Li, J. (2009). A self starting linear multistep method for a direct solution of the general second order Initial value problem. Inter. Journal of Computer Math., 86(5), 817-836.

Kayode, S. J. (2008). An efficient zero-stable numerical method for fourth-order ordinary differential equations. Inter. J. Mathematics and Mathematical Sciences, 1155-1165.

Lambert, J. D. (1973). Computational methods in ordinary differential equation. John Wiley \& Sons Inc. http://dx.doi.org/10.1002/zamm.19740540726

Olabode, B. T. (2009a). A six-step scheme for the solution of fourth order ordinary differential equations. The Pacific Journal of Science and Technology, 10(1).

Olabode, B. T. (2009b). An accurate scheme by block method for third order ordinary differential equations. Journal of Nigerian Association of Mathematical Physics, 10(1), 136-142.

Olabode, B. T., \& Yusuph, Y. (2009). A new block method for special third order ordinary differential equations. Journal of Mathematics \& Statistics Society, 5(3),167-170. http://dx.doi.org/10.1080/00207160500112902

Shampine, L. F., \& Watts, H. A. (1969). Block implicit one step methods. J. of Math of Computation, 23(10), 731-740.

Siamak, M. (2010). A direct variable step block multistep method for solving general third order ODE's. J. of Numer. Algor., 57(1), 53-66.

Majid, Z. A., Azmi, N. A., Suleiman, M., \& Ibrahim, Z. B. (2012). Solving directly general third order ordinary differential equations using two-point four step block method. Sains Malaysiana, 41(5), 623-632.

Zarina, B. I., Mohamed, S., \& lskanla, I. O. (2009). Direct block backward differentiation formulas for solving second order ordinary differential equation. Journal of Mathematics and Computation Sciences, 3(3), 120122. ISSN: 0126-6039. 